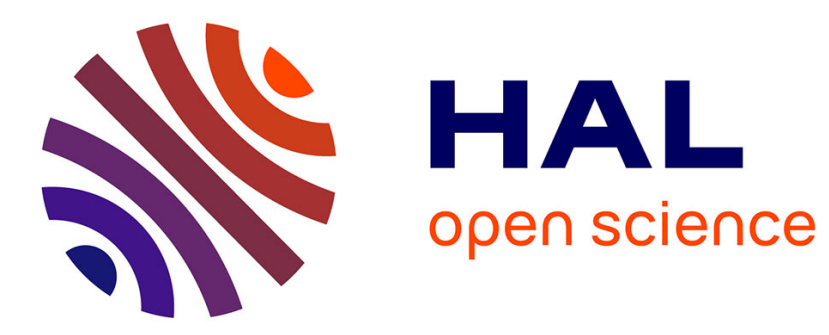

\title{
An Evolutionary Approach for the Optimal Design of the iCub mk.3 Parallel Wrist
}

Raed Bsili, Giorgio Metta, Alberto Parmiggiani

\section{To cite this version:}

Raed Bsili, Giorgio Metta, Alberto Parmiggiani. An Evolutionary Approach for the Optimal Design of the iCub mk.3 Parallel Wrist. IEEE-RAS 18th International Conference on Humanoid Robots (Humanoids 2018), Nov 2018, Beijing, China. hal-01921921

\section{HAL Id: hal-01921921 \\ https://hal.science/hal-01921921}

Submitted on 14 Nov 2018

HAL is a multi-disciplinary open access archive for the deposit and dissemination of scientific research documents, whether they are published or not. The documents may come from teaching and research institutions in France or abroad, or from public or private research centers.
L'archive ouverte pluridisciplinaire HAL, est destinée au dépôt et à la diffusion de documents scientifiques de niveau recherche, publiés ou non, émanant des établissements d'enseignement et de recherche français ou étrangers, des laboratoires publics ou privés. 


\title{
An Evolutionary Approach for the Optimal Design of the iCub mk.3 Parallel Wrist
}

\author{
Raed Bsili ${ }^{+}$, Giorgio Metta*, Alberto Parmiggiani*
}

\begin{abstract}
The payload-to-weight ratio is one of the most important metrics when designing robotic wrists. A solution to maximize it and to reduce the share of the motive power required to drive the robot's links is to employ parallel kinematic mechanisms (PKMs). Indeed PKMs allow relocating distal masses closer to the robot's base actually increasing the overall payload. On the other hand, PKMs are often characterized by limited ranges of motion (RoM) and nonuniform motion in their workspace.

In this article, we considered a class of 2-DOF spherical six-bar mechanisms. We first developed the kinematic model of the system. We then tackled both the workspace limitation and uniformity issues with a numerical optimization approach. Differential evolution (a multi-objective, multivariate, gradientfree optimization method) was applied to the model of the system to explore a large space of parameter combinations.

The optimization algorithm allowed obtaining an almost uniform and large RoM (exceeding $5^{\circ}$ on both axes). We then proceeded with the detailed design of the joint as we envision integrating it on the future releases of the iCub robot forearmhand assemblies.
\end{abstract}

\section{INTRODUCTION}

The payload-to-weight ratio is one of the most important metrics for describing the physical capabilities of robots.

The payload-to-weight ratio of robots with serial kinematics can be improved by combining adjacent joints into multi-DOF parallel kinematics mechanisms (PKMs). Some interesting applications of this design approach can be found in [1], [2], [3] and [4].

PKMs are particularly interesting for medium-size humanoids such as the iCub [5] because of their compact size and good stiffness characteristics. For this purpose, in previous works, we focused on the numerical simulation of N-UU class mechanisms [6] and on their comparison with various closed spherical linkage mechanisms [7].

Indeed, PKMs are often characterized by limited ranges of motion and complex kinematics if compared to serial kinematics manipulators. Moreover, the motor-input/jointoutput Jacobian, i.e. the relation between the motion of the actuators and the resulting joint motion, is often coupled and configuration-dependent. This, in turn, significantly complicates the control of the joint as the same motor motion causes different joint motions in different regions of the mechanism's workspace. Further details about the

\footnotetext{
* Giorgio Metta and Alberto Parmiggiani, are within iCub, Fondazione Istituto Italiano di Tecnologia, Via Morego 30, 16163, Genoa, Italy. giorgio.metta@it.it, alberto.parmiggianidit.it

+ Raed Bsili is within iCub, Fondazione Istituto Italiano di Tecnologia, Via Morego 30, 16163, Genoa, Italy and Danieli telerbotlabs srl, Via Buccari 9, 16153, Genoa, Italy. raed.bsilidit. it
}

characteristics comparison between the PKMs and serial kinematic mechanisms (SKMs) can be found in [8].

When designing a manipulator, especially a PKM, an interesting metric to evaluate the "uniformity" of motion in the mechanism's workspace is isotropy, as defined in [9].

In this article, we investigate the 2DOF 2DSPM parallel mechanism introduced by Ogata et al. [10] and later refined by Ueda et al. in [11].

The mechanism's RoM and the degree of variations in the motor-input/joint-output Jacobian are a function of the design parameters. We addressed both aspects by selecting the design parameters with a methodology based on the differential evolution numerical optimization method.

The article describes in detail the approach followed and presents the definition and solutions of the optimization process. In addition, we present qualitative and quantitative analysis on the effect of the design parameters on the wrist mechanism behavior.

\section{APPROACH}

The current iCub mk. 3 wrist RoM requirements are $\pm 56^{\circ}$ and $\pm 38^{\circ}$ on the pitch and yaw axes. The goal we pursued in this work was to design a 2DSPM with equivalent and possibly larger range of motion and with a uniform mechanism behaviour. The Fig. 1 presents the design model (at zeroposition) of the 2DSPM where the green part defines the endeffector. The pitch rotation $\phi_{p}$ is defined as the end-effector's

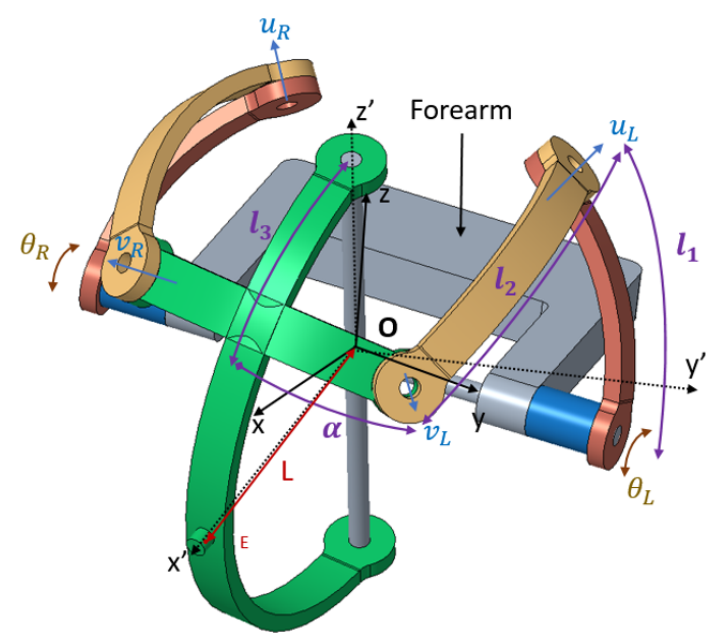

Fig. 1: CAD model of the investigated 2DSPM

rotation about the $y$-axis while yaw as the end-effector's rotation $\phi_{y}$ about the $z$-axis. The distance between the endeffector point $E$ and the center of rotation $O$ denoted by $L$, is 
the scale of the wrist. The design configuration is described by the design parameters, denoted by $l_{1}, l_{2}, l_{3}$ and $\alpha$, that define the wrist's links geometry. There are two actuated joints: a left and a right one, denoted respectively by $\theta_{L}$ and $\theta_{R}$. To achieve an optimal design, a proper mathematical model describing completely the kinematics in function of the design parameters was modeled. Then, the optimization model describing the optimal design configuration from mechanism uniformity and workspace width point of view was set up and solved. To generate the workspace, a square grid input to the actuated joints with a given amplitude $I$ is generated, resulting in the generation of all different input combinations of $\theta_{L}, \theta_{R} \in[-I, I]$ range. In this paper, the square grid semi-length $I$ is called the amplitude.

\section{A. Kinematics}

A fixed coordinate frame $O(x, y, z)$ in the center of rotation $O$ is placed with the same origin of the end-effector coordinate frame $O\left(x^{\prime}, y^{\prime}, z^{\prime}\right)$ as shown in Fig. 1 . The vectors $u_{L}$ and $v_{L}$ describing the rotation axis of the passive joints of the left leg are expressed using the rotational matrix $R_{\text {axis }}($ angle $) \in S O(3)$ :

$$
\begin{gathered}
u_{L}=R_{y}\left(\theta_{L}\right) \cdot R_{x}\left(l_{1}\right) \cdot[0,1,0]^{T} \\
v_{L}=R_{y}\left(\phi_{p}\right) \cdot R_{z}\left(\phi_{y}-\frac{\pi}{2}\right) \cdot R_{x}\left(\frac{\pi}{2}-l_{3}\right) \cdot R_{z}(\alpha) \cdot[0,1,0]^{T}
\end{gathered}
$$

where $S O(3)$ denotes the $3 D$ rotation group. The design parameter $l_{2}$ imposes the following mathematical constraint:

$$
u_{L} \cdot v_{L}-\cos \left(l_{2}\right)=0
$$

The same mathematical operations have been applied on the right leg. Thus, the kinematics problem became written as a 2-dimensional system of highly non-linear equations, with two unknowns $\phi_{p}$ and $\phi_{y}$ to solve the forward kinematics:

$$
\begin{aligned}
& f_{L}=u_{L}^{T} \cdot v_{L}-\cos \left(l_{2}\right) \\
& f_{R}=u_{R}^{T} \cdot v_{R}-\cos \left(l_{2}\right)
\end{aligned}
$$

This system is solved using an optimization algorithm meant to minimize the sum square of $f_{L}$ and $f_{R}$. Due to the theoretical non-unicity of solutions, mathematical constraints were added to ensure the uniqueness of the realistic solution. Therefore, the forward kinematics are reduced to the following constrained and bounded optimization problem, with a dimension $N=2$ :

$$
\underset{x=\left[\phi_{p}, \phi_{y}\right]}{\operatorname{argmin}}\left\|f(x)=f_{L}^{2}+f_{R}^{2}\right\|
$$

where:

$$
\phi_{p}, \phi_{y} \in\left[-180^{\circ}, 180^{\circ}\right]
$$

and:

$$
\left\{\begin{array}{l}
g_{L}(x)=[0,1,0] \cdot\left(u_{L} \times v_{L}\right) \geqslant 0 \\
g_{R}(x)=[0,1,0] \cdot\left(u_{R} \times v_{R}\right) \geqslant 0
\end{array}\right.
$$

The optimization problem has been solved numerically by providing the exact analytic expressions of the objective function's Hessian $H_{f}$ and the Jacobian of both the objective and constraints functions $J_{f}$ and $J_{g}$ :

$$
\begin{gathered}
J_{f}=\left[\frac{d f}{d \phi_{p}}, \frac{d f}{d \phi_{y}}\right] \in \mathbb{R}^{1 \times 2} \\
H_{f}=J(\nabla f)^{T} \in \mathbb{R}^{2 \times 2}
\end{gathered}
$$

The inverse kinematics are solved in the same way described above by solving $x=\left[\theta_{L}, \theta_{R}\right]$, for a desired pitch and yaw $\left[\phi_{p}, \phi_{y}\right]$ configuration.

\section{B. The Global Isotropy and Workspace Numbers}

An important criterion to evaluate a parallel manipulator is to evaluate its mechanism uniformity using a metric called isotropy, denoted by $\Delta$. It is a measure that indicates how well over the workspace a wrist is manipulable and how close or far it is from singularity at the same time. The isotropy $\Delta$ is defined as:

$$
\Delta(J)=\frac{\sqrt[m]{\operatorname{det}\left(J J^{T}\right)}}{t r\left(J J^{T}\right) / m} \in[0,1]
$$

where $J \in \mathbb{R}^{m \times n}$ is the manipulator Jacobian. The isotropy could be written equivalently in terms of the $J J^{T}$ eigenvalues, denoted by $\left\{\lambda_{i}\right\}_{i \in \mathbb{N}}$ :

$$
\Delta(J)=\frac{\left(\prod_{i=1}^{m} \lambda_{i}\right)^{1 / m}}{\left(\sum_{i=1}^{m} \lambda_{i}\right) / m} \in[0,1]
$$

where $m$ denotes the number of DoF of a manipulator's end-effector. In our case, $m=2$. The isotropy's numerator and denominator are defined respectively as the geometric mean, and the arithmetic mean of the $J J^{T}$ eigenvalues. The geometrical mean describes the wrist manipulability. When $\Delta=0$, the manipulator is in a singular configuration (i.e. $\left.\exists \quad i \in \mathbb{N}, \quad \lambda_{i}=0\right)$. Using the AM-GM Inequality, one can conclude that the isotropy is indeed bounded $\in[0,1]$ as $J J^{T}$ is positive definite. Further, $\Delta$ is maximum, only, and only if all the manipulator's Jacobian eigenvalues are non-zeros and are equal to each other, implying the full Jacobian $m$ - spheroid which we intend to realize i.e. a perfectly posed Jacobian matrix. The isotropy is a local property as it depends on the end-effector pose. Therefore, we evaluate the isotropy over the whole workspace with its weighted mean:

$$
\bar{\Delta}=\frac{\int_{W} \Delta(J) d W}{\int_{W} d W} \in[0,1]
$$

where $W$ denotes implicitly the workspace area spanned by the end-effector's orientation angles $\phi_{p}$ and $\phi_{y}$. In this work, we loosely refer to $\bar{\Delta}$ as the Global Isotropy Number. It is a metric used to compare the uniformity of different manipulators mechanisms, independently from their scale $L$. Another measure of motion uniformity commonly used in the literature is the Global Conditioning Index, introduced by Gosselin and Angeles in [12], which is a measure of the Jacobian condition number over the whole workspace. The condition number of the Jacobian matrix has been 
first introduced in [13] and it is used as well as a metric to describe how ill-posed a matrix is. In this work, we nevertheless preferred to use the measure of isotropy, denoted by $\Delta$, for the following reasons:

- The $\Delta$-index is faster to compute numerically than the condition number. This could be explained by the fact that the condition number requires computing the matrix inverse, which is relatively more computationally expensive.

- The $\Delta$-index, unlike the condition number, can be expressed analytically in function of the manipulator's joints angles. This speeds up further its computation.

In order to evaluate the workspace width of the manipulator and compare it to other manipulators, independently from their scale $L$, we propose the following Workspace Number:

$$
\bar{\Omega}=\frac{\int_{W} d W}{S_{J}}=\frac{\int_{\phi_{p_{\min }}}^{\phi_{p_{\max }}} \int_{\phi_{y_{\min }}}^{\phi_{y_{\max }}} d \phi_{y} d \phi_{p}}{\int_{\theta_{L_{\min }}}^{\theta_{L_{\max }}} \int_{\theta_{R_{\min }}}^{\theta_{R_{\max }}} d \theta_{L} d \theta_{R}} \in \mathbb{R}_{\geqslant 0}
$$

where here $S_{J}$ denotes the area spanned by the input actuated joints $\theta_{R}$ and $\theta_{L}$. When the input is a square grid with an amplitude $I$, as in our case, the joints angular workspace area denoted by $S_{J}$ becomes simplified as $S_{J}=$ $4 I^{2}$. More details are explained in Fig. 3 .

For example, in the case of an "ideal" gimbal, $\bar{\Delta}=\bar{\Omega}=1$. Finally, the mapped cartesian workspace area denoted by $S_{C}$ is defined as the area of the end-effector workspace envelope (see Appendix).

\section{Optimization}

The optimal design here requires finding the best design parameters that ensure the maximum isotropy and the widest workspace. The maximization of the isotropy and the workspace was expressed as an $N=4$ dimensional optimization problem:

$$
\underset{X=\left[l_{1}, l_{2}, l_{3}, \alpha\right]}{\operatorname{argmin}}\left\|f(X)=1-\left(\Delta_{\min } \cdot \bar{\Delta} \cdot \Delta_{\max }\right) \cdot \bar{\Omega}\right\|
$$

where:

$$
l_{1}, l_{2}, l_{3}, \alpha \in\left[9^{\circ}, 85^{\circ}\right]
$$

and:

$$
g(X)=S_{l_{1}} S_{\alpha}-\left(C_{l_{2}}-S_{\alpha} C_{l_{1}}\right) \geqslant 0
$$

In which the above abbreviations are $S_{x}=\sin (x)$ and $C_{x}=$ $\cos (x)$. In our case, the Workspace Number has been restricted to be bounded i.e. $\bar{\Omega}(X) \in[0,1]$. The above bounds have been added to ensure a realizable potential design. The above constraint $g(X)$ has been added to ensure that the design parameters describe a design where the zero pitch and zero yaw positions of the manipulator are defined from a symmetric zero position of the actuated joints. Due to the complexity of the objective function $f(X) \in \mathbb{R}$, and the errorprone numerical approximation of its Jacobian $J_{f} \in \mathbb{R}^{1 \times 4}$, a gradient-free optimization technique is required. Therefore, meta-heuristic evolutionary optimization algorithms were needed and the Differential Evolution $D E$ [14] optimizer is selected. initialize the first generation of individuals;

$\mathrm{G}=1$

while Stop criterion not met do

for each target vector $X_{\mathrm{i}, \mathrm{G}}$;

for $i=1$ to $N P$ do

Mutation: generate a donor vector $V_{\mathrm{i}, \mathrm{G}}$;

$V_{\mathrm{i}, \mathrm{G}}=X_{\mathrm{ri} 1, \mathrm{G}}+F\left(X_{\mathrm{ri} 2, \mathrm{G}}-X_{\mathrm{ri}, \mathrm{G}}\right)$

for $j=1$ to $N$ do

Crossover: generate a trial vector $u_{\mathrm{i}, \mathrm{G}}$;

$$
u_{\mathrm{j}, \mathrm{i}, \mathrm{G}}=\left\{\begin{array}{l}
\mathrm{V}_{\mathrm{j}, \mathrm{i}, \mathrm{G}}, \text { if } U_{j}(0,1) \leqslant C R \text { or } j=j_{\mathrm{rnd}} \\
\mathrm{X}_{\mathrm{j}, \mathrm{i}, \mathrm{G}}, \quad \text { otherwise }
\end{array}\right.
$$

end

Selection: accept the trial vector if not worse than the target vector;

$X_{\mathrm{i}, \mathrm{G}+1}= \begin{cases}\mathrm{U}_{\mathrm{i}, \mathrm{G}}, & \text { if } \\ \mathrm{X}_{\mathrm{i}, \mathrm{G}}, & f\left(U_{\mathrm{i}, \mathrm{G}}\right) \leqslant f\left(X_{\mathrm{i}, \mathrm{G}}\right) \\ \text { otherwise }\end{cases}$

Algorithm 1: Differential Evolution pseudo-code

The $D E$ optimization algorithm requires three parameters to tune: the mutation factor $F \in[0,2]$, the crossover probability $C R \in[0,1]$ and the population size NP. Algorithm. 1 presents the $D E$ pseudo-code where a rand/1/bin strategy was used to generate the donor vector [15], in which $U(a, b) \in \mathbb{R}$ denotes a random number between $a$ and $b$. The residual, defined as well as the candidate energy, is denoted by $f(X) \in \mathbb{R}, \quad X \in \mathbb{R}^{N}$.

The algorithm works by iterating over every generation $G$ and executing mainly three phases: mutation, crossover and finally the selection phase.

\section{Computational Perspectives}

To compute the Global Isotropy Number of a design parameters set, first, the forward kinematics over the whole workspace are solved using the optimization problem described by Eq. (6). Secondly, the Jacobian matrix $J$ and the local isotropy are calculated by feeding the forward kinematics information to the analytic expressions of $J\left(\left[\theta_{R}, \theta_{L}\right],\left[\phi_{p}, \phi_{y}\right]\right)$ and $\Delta(J)$. Lastly, the Global Isotropy Number and Workspace Number are computed numerically using Eq. (11) and Eq. (12), respectively. All analytic expressions were derived using symbolic computational methods. The $D E$ optimization algorithm allowed to explore a significant amount of parameter combinations. To make a ballpark comparison, a brute force exploration of the parameter set with a $1^{\circ}$ resolution would have required more than 5000 times the computations that were performed for this study. The whole algorithm is developed in the Python programming language [16]. All computations have been executed on a Dual AMD Opteron computer server with a $6328 @ 3.2 \mathrm{GHz}$ processor and 64 GB RAM. 


\section{RESULTS}

In the beginning, a square grid input with an amplitude $I=65^{\circ}$ was used. After tuning the $D E$ optimizer parameters, the crossover factor was set to $C R=0.1$ to have a more stable candidates population. The mutation factor was taken arbitrary from $F=U(1.5,1.9)$ to randomize it for every generation. This increased the search radius at the cost of a slower convergence rate. Population size is set to $N P=60$ candidate individuals. To initialize the first population of individuals, a Latin Hypercube Sampling (LHS) method [17] was used to maximize the coverage of the available parameters space i.e. $\mathbb{R}^{4}$. Finally, a best/l/bin strategy has been selected to formulate the donor vector gene, taking the best candidate to mutate instead of a random individual vector $X_{\text {ril,G. }}$.

As a result, the optimum design parameters vector was found to be $\mathbf{O P T}_{\mathbf{1}}=\left[\mathbf{3 3 . 7}^{\circ}, \mathbf{8 3}^{\circ}, \mathbf{3 2 . 7}^{\circ}, \mathbf{1 0 . 7}^{\circ}\right]$, whose isotropy plot in the $Y Z$-plane is shown in Fig. 2. All plots were performed using a normalized scale $L=1$, so that all the results are bounded between -1 and 1 . Furthermore, all colorbars are scaled equally from the corresponding $\Delta_{\min }$ to $\Delta_{\max }$, in order to help the reader appreciate the differences between the isotropy distributions for the different design parameters candidates. The design parameters vector $O P T_{1}$ is shown

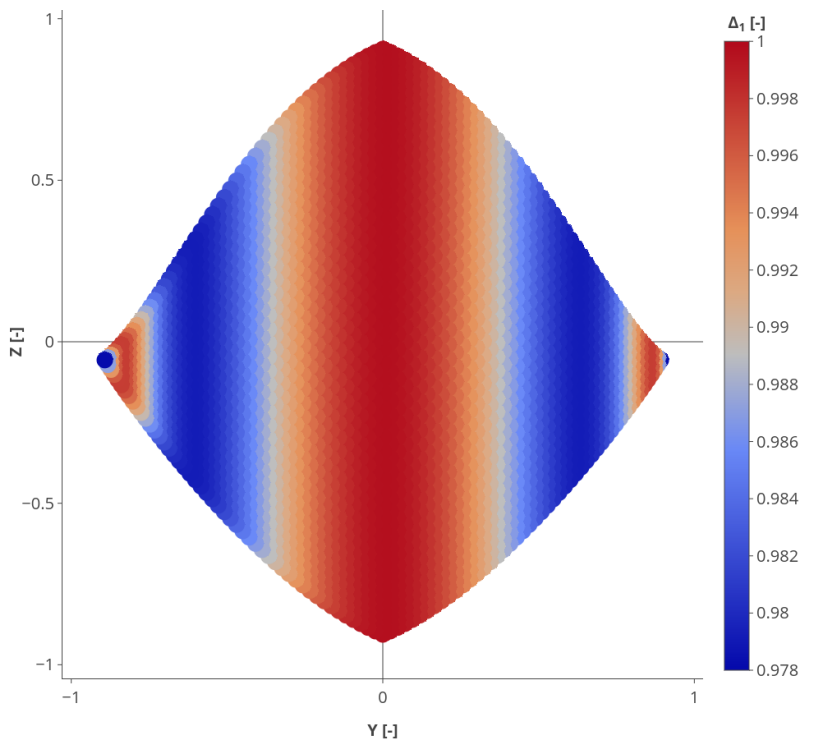

Fig. 2: $O P T_{1} 2 \mathrm{D}$ isotropy contour on $Y Z$-plane $\left(I=65^{\circ}\right)$

performing a full isotropic spheroid where $\left(\Delta_{\min }, \bar{\Delta}, \Delta_{\max }\right)=$ $(0.978,0.998,1)$ and with rotational ranges consisting in a pitch: $\phi_{p} \in\left[-65^{\circ}, 65^{\circ}\right]$ and a yaw: $\phi_{y} \in\left[-63.1^{\circ}, 63.1^{\circ}\right]$. This parameter combination yields the required ranges, as shown in Fig. 3, and improves the design parameters $\mathbf{P}=\left[\mathbf{4 2}^{\circ}, \mathbf{7 4}^{\circ}, \mathbf{5 0}^{\circ}, \mathbf{2 0 . 4}^{\circ}\right]$ proposed by Ueda et al. in [11]. The Workspace Number $\bar{\Omega}$ was computed as the contour angular area mapped by the end-effector's angles, divided by the square grid input area defined by the black rectangle, as shown in Fig. 3. Extending the input square grid to an amplitude $I=80^{\circ}$ causes the $O P T_{1}$ isotropy to drop significantly to $\Delta_{\min }=0.11$, which makes it not the best

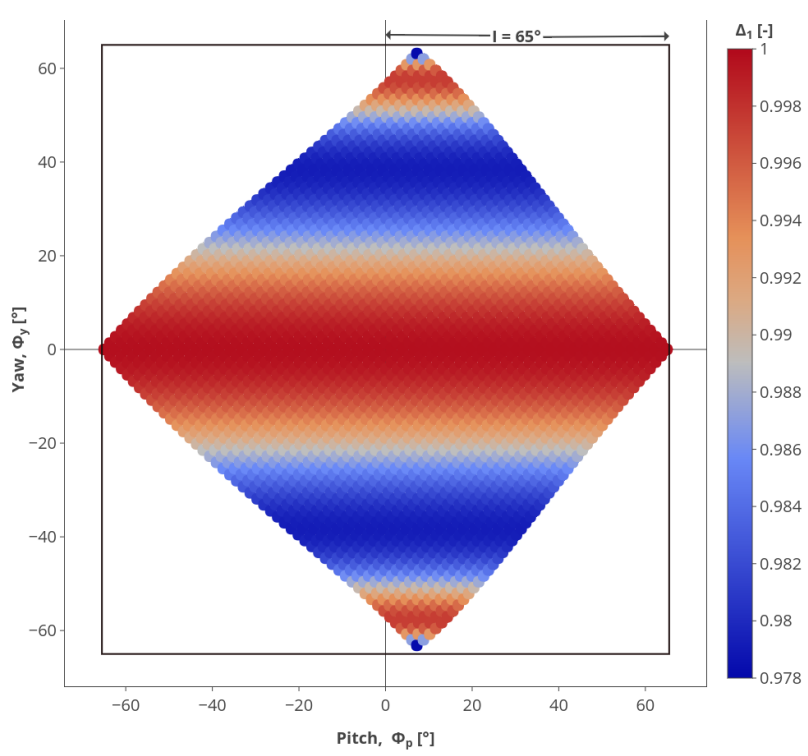

Fig. 3: $O P T_{1} 2 \mathrm{D}$ isotropy contour on the Euler plane $\left(I=65^{\circ}\right)$

solution, from isotropy point of view. The optimization process has been adjusted to optimize only the isotropy because an input of $I \geqslant 65^{\circ}$ is enough to fulfill the iCub mk.3 ranges of motion requirement.

As a result, the optimum $\mathbf{O P T}_{\mathbf{2}}=\left[\mathbf{4 1}^{\circ}, \mathbf{7 6 . 3}^{\circ}, \mathbf{4 1 . 1}^{\circ}, \mathbf{2 5 . 7 ^ { \circ }}\right]$, (see Fig. 4) was found to be the best solution scoring a $\left(\Delta_{\min }, \bar{\Delta}, \Delta_{\max }\right)=(0.88,0.97,1)$. It is performing a nearly full isotropic spheroid, improving $O P T_{1}$ and design parameters $P$ plotted in Fig. 6. In Fig. 5, we show the $2 D$ isotropy

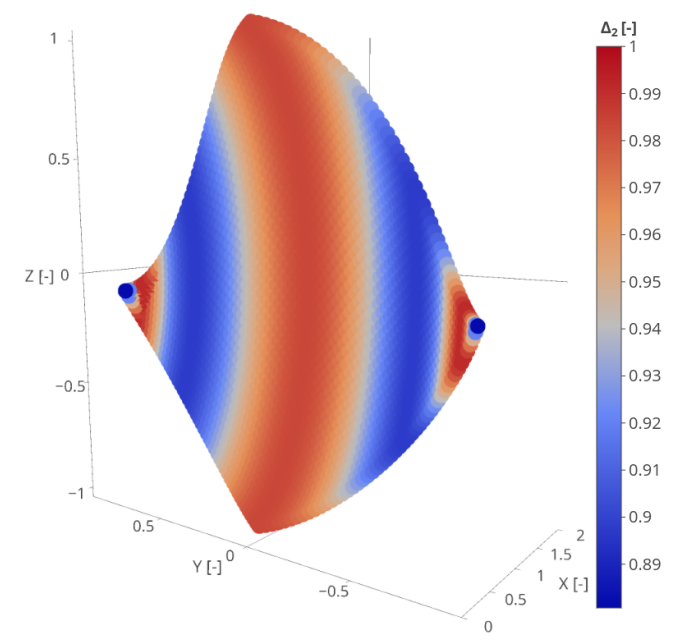

Fig. 4: $O P T_{2} 3 D$ Isotropy on the cartesian workspace $(I=$ $80^{\circ}$ )

contour of $O P T_{1}$ design parameter on $Y Z$-plane overlapped by the workspace of $O P T_{2}$. As shown in Fig. 5, the $O P T_{2}$ has thinner workspace than $O P T_{1}$, but better isotropy distribution due to the considerable $O P T_{1}$ isotropy drop in the workspace lateral corners. This means that in such design configuration, approaching these regions would result in a significant amplification of the end-effector velocity when compared to other regions in the workspace. This is a behavior to avoid 


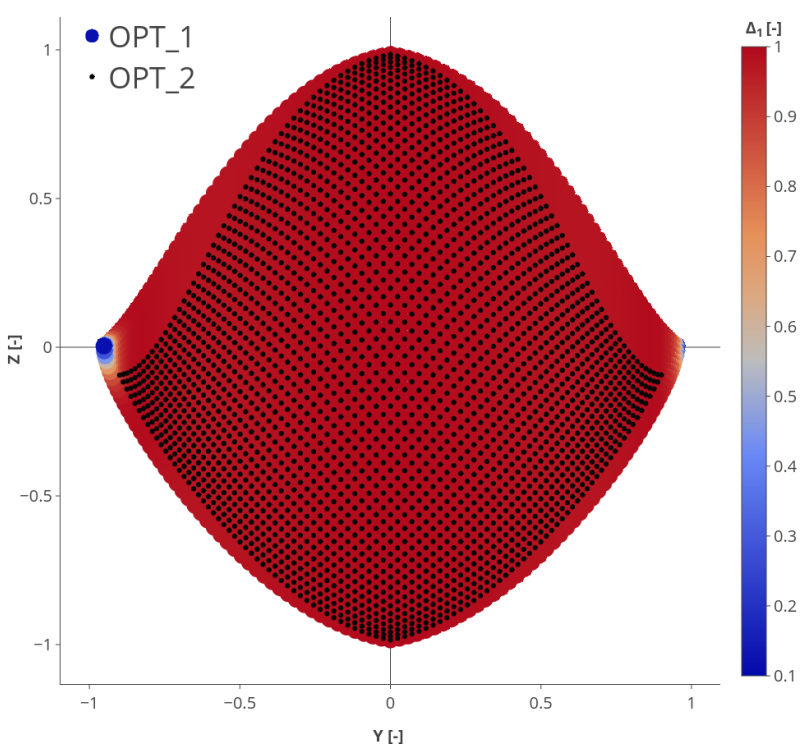

Fig. 5: $O P T_{2}-O P T_{1} 2 \mathrm{D}$ isotropy and workspace comparison $\left(I=80^{\circ}\right)$

to ensure more robust control of the wrist.

To validate the results, using PTC Creo Parameteric 4.0, a

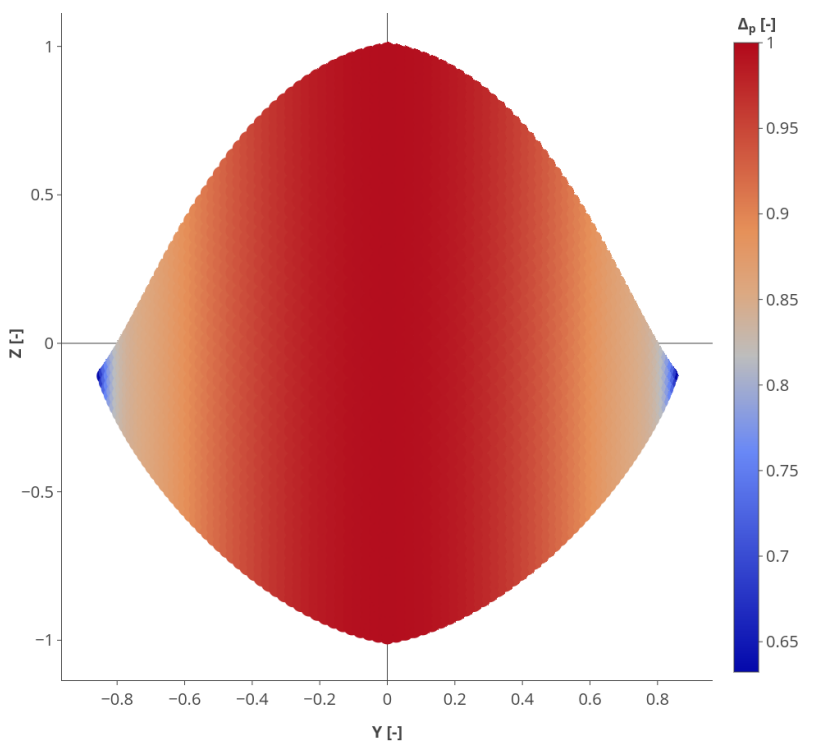

Fig. 6: $P$ - 2D isotropy contour on $Y Z$-plane $\left(I=80^{\circ}\right)$

CAD model with the design parameters $O P T_{1}, O P T_{2}$ and $P$ were modeled. A simulation with the same square grid input $I$ has been performed, showing an approximate matching between the mathematical model and the CAD simulation with an average error equal to $\bar{\varepsilon} \sim 10^{-5}$ on the isotropy $\Delta$ (see Fig. 7).

\section{DISCUSSION}

To increase the likelyhood of obtaining global optimality, different optimization runs were performed over $G=100$ generations. The optimizer converged in all the trials to the same optimum, as shown in Fig. 8. The optimization runs

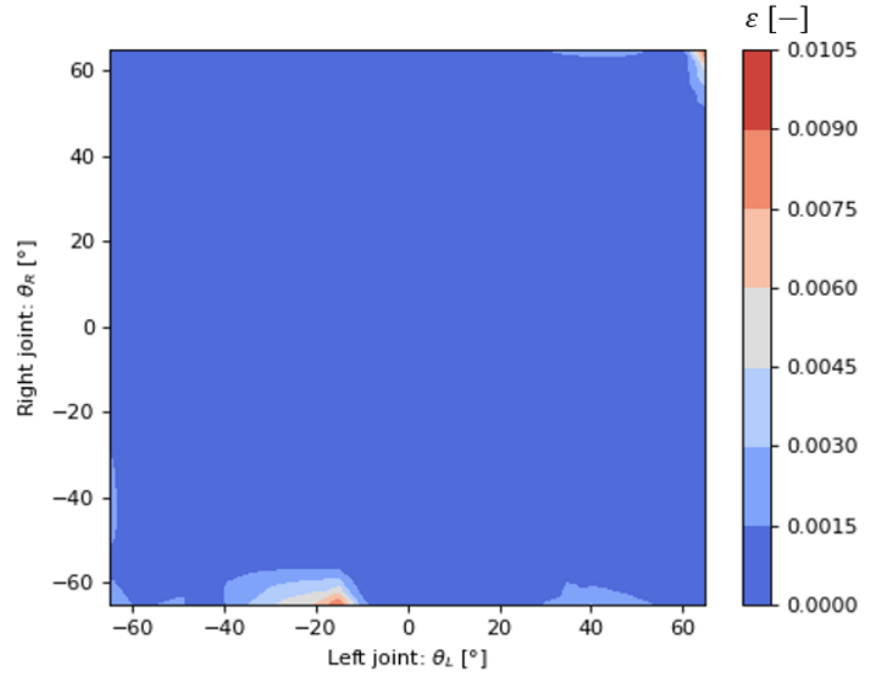

Fig. 7: $O P T_{1}$ Isotropy comparison between anatylical and CAD models: $\varepsilon=\left|\Delta_{\text {Creo }}-\Delta\right|,\left(I=65^{\circ}\right)$

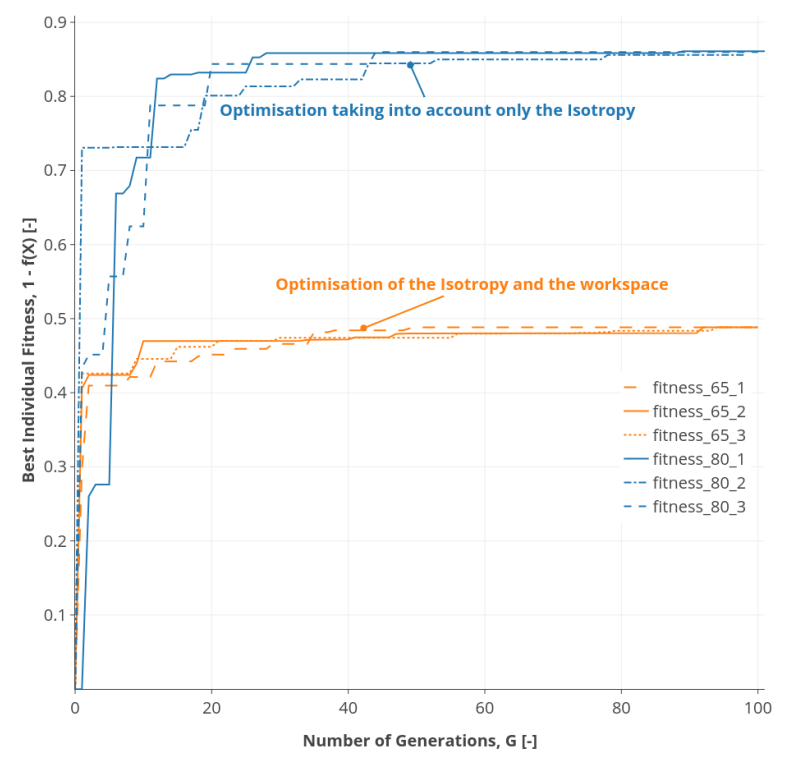

Fig. 8: Convergence of the fittest individual per generation G

were all performed at the same time, in parallel, and on the same computer server. The computations for the six trials required approximately seven days.

One can notice that such a 2DSPM is characterized by always having a symmetric ranges of motion, a constant isotropy along the pitch direction, and a variable symmetric isotropy along the yaw direction. In particular, $O P T_{1}$ and $O P T_{2}$ designs isotropies tend to oscillate along the yaw direction with variable amplitudes, however, $P$ design isotropy tends to decrease along it, as shown in Fig. 9. This is mathematically consistent with the fact that we have always $\left|\phi_{p}\right|_{\max }=I$, as shown in Tab I. This is interpreted by the fact that an angular variation on the actuated joints would induce the same angular variation on the end-effector's pitch angle, implying a perfectly posed Jacobian matrix along the pitch 


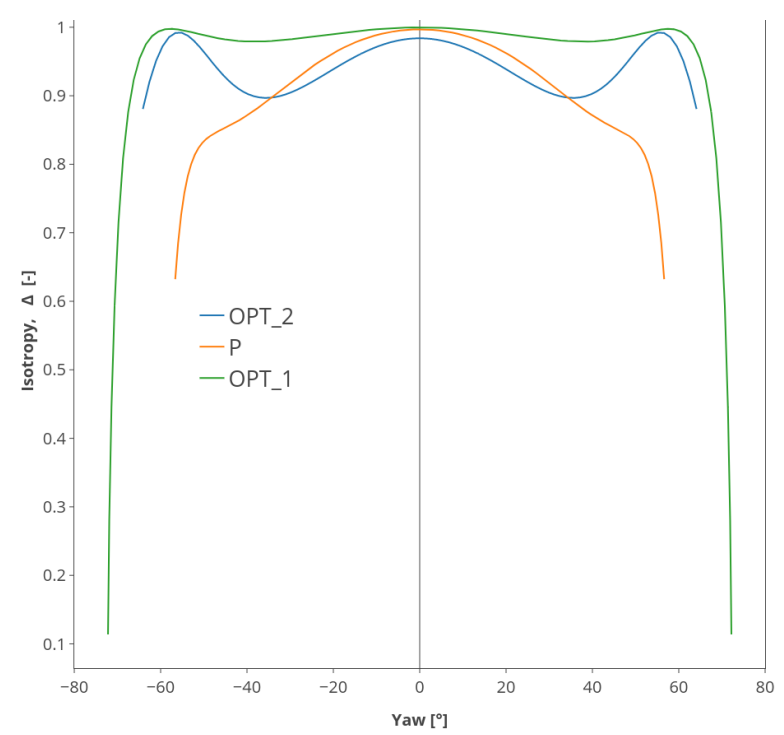

Fig. 9: Isotropy variation along yaw direction $\left(I=80^{\circ}\right)$

direction, i.e. $\quad \Delta(J) \simeq 1$.

It should be noted that tuning the input amplitude $I$ would result in finding different optima. This depends on how the optimum was defined, from isotropy or workspace width point of view, or both of them. For example, during the optimization process, we count several candidates found to have a wider workspace than $O P T_{2}$, but lower Global Isotropy Number. Alternative loss functions could be used to define the objective function $f(X)$ in order to speed up the convergence rate and control the optimization process. In the end, $O P T_{1}$ is shown to be likely the global optimum when $I=65^{\circ}$, validating the iCub mk.3 wrist range of motion requirement. At last, for general applications, in which wider ranges are required, for example, $I=80^{\circ}, O P T_{2}$ is recommended to use as design parameters. The scatter matrix

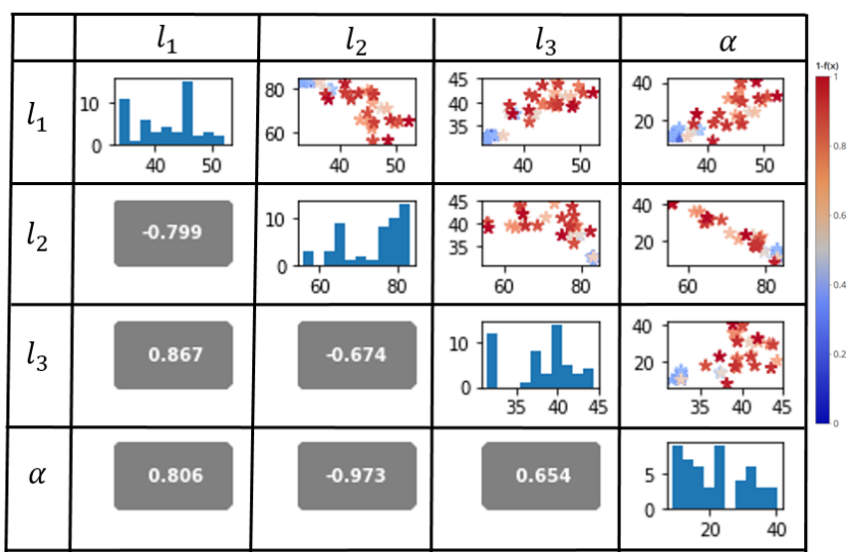

Fig. 10: Scatter matrix for the objective functions and the design parameters

shown in Fig. 10 presents the inter-dependencies between the decision variables during the optimization process, when $I=65^{\circ}$, obtained from the Pareto front, by sorting the best 50 individuals. It is shown that during optimization, through successive generations, the parameters $\alpha$ and $l_{2}$ have evolved inversely proportional to each other, with a correlation factor equal to -0.973 . Table II summarizes the different design parameters candidates that were discussed above. Table I

TABLE I: Comparison of different design parameters

\begin{tabular}{|c|c|c|c|}
\hline $\mathbf{I}=\mathbf{6 5}^{\circ}$ & $\left(\Delta_{\min }, \bar{\Delta}, \Delta_{\max }\right)$ & $\left(\bar{\Omega}, S_{C}[-]\right)$ & $\left(\left|\phi_{p}\right|_{\max },\left|\phi_{y}\right|_{\max }\right)$ \\
\hline $\mathbf{O P T}_{\mathbf{1}}$ & $(0.978,0.998,1)$ & $(0.5,4.92)$ & $\left(65^{\circ}, 63.1^{\circ}\right)$ \\
\hline $\mathbf{O P T}_{\mathbf{2}}$ & $(0.90,0.97,1)$ & $(0.41,4.17)$ & $\left(65^{\circ}, 50.6^{\circ}\right)$ \\
\hline $\mathbf{P}$ & $(0.80,0.95,1)$ & $(0.41,4.15)$ & $\left(65^{\circ}, 49.3^{\circ}\right)$ \\
\hline $\mathbf{I}=\mathbf{8 0 ^ { \circ }}$ & & & \\
\hline $\mathbf{O P T}_{\mathbf{1}}$ & $(0.11,0.92,1)$ & $(0.49,6.58)$ & $\left(80^{\circ}, 72.5^{\circ}\right)$ \\
\hline $\mathbf{O P T}_{\mathbf{2}}$ & $(0.88,0.97,1)$ & $(0.41,6.02)$ & $\left(80^{\circ}, 64^{\circ}\right)$ \\
\hline $\mathbf{P}$ & $(0.63,0.93,1)$ & $(0.39,5.72)$ & $\left(80^{\circ}, 56^{\circ}\right)$ \\
\hline
\end{tabular}

summarizes the results found so far and their comparison. Finally, one notes that the dynamics behavior of such 2DSPM

TABLE II: Design parameters candidates

\begin{tabular}{|c|c|}
\hline & {$\left[l_{1}, l_{2}, l_{3}, \alpha\right]$} \\
\hline $\mathbf{O P T}_{\mathbf{1}}$ & {$\left[33.7^{\circ}, 83^{\circ}, 32.7^{\circ}, 10.7^{\circ}\right]$} \\
\hline $\mathbf{O P T}_{\mathbf{2}}$ & {$\left[41^{\circ}, 76.3^{\circ}, 41.1^{\circ}, 25.7^{\circ}\right]$} \\
\hline $\mathbf{P}$ & {$\left[42^{\circ}, 74^{\circ}, 50^{\circ}, 20.4^{\circ}\right]$} \\
\hline
\end{tabular}

would be similarly isotropic as its kinematics behavior. This is justified by the fact that, when neglecting gravitation and frictions, the task force space is mapped to the joints force space by $J^{T}$ and that $\Delta(J)=\Delta\left(J^{T}\right)$, implying a robust hybrid control of such wrist.

\section{DESIGN}

The $O P T_{1}$ design parameters were selected for the iCub mk.3 wrist, and used for the preliminary assembly design represented in Fig. 11. Because of the small size of the

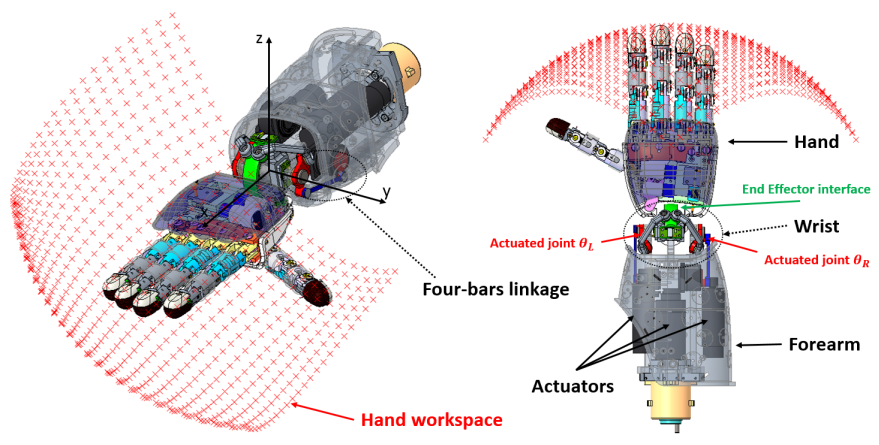

Fig. 11: $\left(O P T_{1}\right)$ - iCub mk.3 hand and forearm preliminary design

iCub forearm, a Four-bars linkage was selected to actuate the active joints $\theta_{L}$ and $\theta_{R}$. One of the main advantages of using the planar Four-bars linkages is to ensure an efficient power transmission between the two joints (crank and follower) while keeping the assembly relatively simple and compact. On the other hand, the Four-bars linkage has a singular configuration (when all linkages are aligned) that shall be avoided. This limitation needs to be taken into account since passing through the singular configuration shall be avoided in all wrist configurations. In order to avoid the singularities 


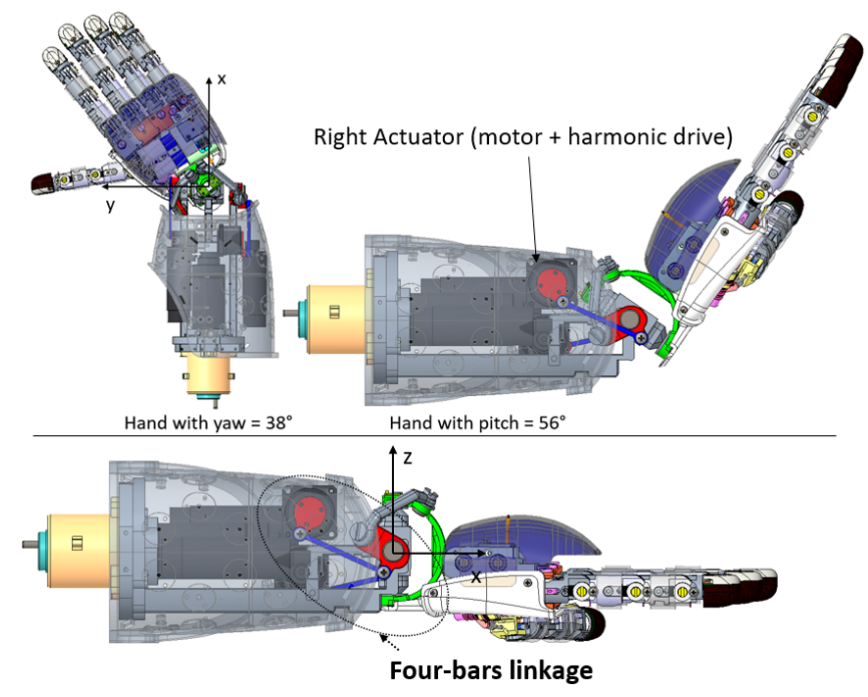

Fig. 12: $\left(O P T_{1}\right)$ - iCub mk.3 hand reaching its required ranges of motion

TABLE III: Design's range of motion summary

\begin{tabular}{|l|l|l|}
\hline & Pitch $\left[{ }^{\circ}\right]$ & Yaw $\left[^{\circ}\right]$ \\
\hline iCub mk.3 Wrist RoM Requirement & \pm 56 & \pm 38 \\
\hline$O P T_{1}$ Theoretical RoM & \pm 65 & \pm 63.1 \\
\hline$O P T_{1}$ Mechanical RoM & \pm 58 & \pm 50 \\
\hline
\end{tabular}

and ensure that the follower moves inside a RoM of an amplitude $I= \pm 65^{\circ}$, an offset angle has been given to it and to the crank. The two active DoF are actuated by two Faulhaber 1724T012 DC motors coupled to two CSF-5-30$2 H X-F-1$ Harmonic Drive compact speed reducers. To avoid mechanical interferences and collisions among some design elements, we have constrained our mechanism to have a range of motion RoM consisting of \pm 58 on the pitch and \pm 50 on the yaw axis. Table III summarizes the design ranges of motion. Finally, Fig. 12 shows the iCub mk.3 wrist reaching $56^{\circ}$ on the pitch axis and $38^{\circ}$ on the yaw axis, fulfilling the required iCub mk.3 wrist range of motion requirement.

\section{CONCLUSIONS}

A full mathematical model has been designed to ensure the global optimal design of a 2DSPM wrist from isotropy and workspace point of view. All models have been tested on PTC Creo Parameteric 4.0 in which a simulation was performed to validate the results. The simulation showed a smooth and realistic motion within the input range's amplitude $I$, with results that match the developed mathematical model with an $\bar{\varepsilon} \sim 10^{-5}$ average error on the isotropy.

Finally, the $O P T_{1}$ design has been selected for rapid prototyping to test it on the iCub mk.3 forearm, as shown in Fig. 11. The selected wrist design has shown promising results with a maximum attained isotropy $\bar{\Delta} \simeq 1=\Delta_{\max }$, over a symmetric workspace $\left(\left|\phi_{p}\right|_{\max },\left|\phi_{y}\right|_{\max }\right)=\left(65^{\circ}, 63.1^{\circ}\right)$.

\section{APPENDIX}

The wrist Jacobian $J \in \mathbb{R}^{2 \times 2}$ was computed as $J=-J_{\phi}^{-1} J_{\theta}$ where:

$$
f=\left[f_{L}, f_{R}\right] ; \quad J_{\theta}=\frac{\partial f}{\partial \theta} ; \quad J_{\phi}=\frac{\partial f}{\partial \phi}
$$

In which $\theta=\left[\theta_{L}, \theta_{R}\right]$ and $\phi=\left[\phi_{p}, \phi_{y}\right]$. Once the forward kinematics solved, the end-effector cartesian position described by the point $E$ coordinates on the base coordinate frame $O(x, y, z)$ was found in function of the wrist scale $L$ and the solved Euler angles:

$$
\left\{\begin{array}{l}
x_{E}=L \cdot \cos \left(\phi_{p}\right) \cdot \cos \left(\phi_{y}\right) \\
y_{E}=L \cdot \sin \left(\phi_{y}\right) \\
z_{E}=-L \cdot \sin \left(\phi_{p}\right) \cdot \cos \left(\phi_{y}\right)
\end{array}\right.
$$

The mapped cartesian workspace area $S_{C}$ was then computed by integrating over the $x_{E}, y_{E}$ and $z_{E}$ values.

\section{ACKNOWLEDGMENT}

This study was conducted as a part of the Deictic Communication (DComm) project and has received funding from the European Union's Horizon 2020 research and innovation programme under the Marie Skodowska-Curie Actions grant agreement No 676063.

\section{REFERENCES}

[1] J. Sofka, V. Skormin, V. Nikulin, and D. Nicholson, "Omni-Wrist III- a new generation of pointing devices. Part I: Laser beam steering devices- mathematical modeling," IEEE Transactions on Aerospace and Electronic Systems, vol. 42, pp. 718-725, Apr. 2006.

[2] F. L. Hammond, R. D. Howe, and R. J. Wood, "Dexterous highprecision robotic wrist for micromanipulation," in Advanced Robotics (ICAR), 2013 16th International Conference on, pp. 1-8, IEEE, Nov. 2013.

[3] A. V. Sureshbabu, J. H. Chang, L. Fiorio, A. Scalzo, G. Metta, and A. Parmiggiani, "A parallel kinematic wrist for the R1 humanoid robot," in 2017 IEEE International Conference on Advanced Intelligent Mechatronics (AIM), pp. 1215-1220, IEEE, July 2017.

[4] G. Wu, S. Caro, S. Bai, and J. Kepler, "Dynamic modeling and design optimization of a 3-dof spherical parallel manipulator," Robotics and Autonomous Systems, vol. 62, no. 10, pp. 1377 - 1386, 2014.

[5] A. Parmiggiani, M. Maggiali, L. Natale, F. Nori, A. Schmitz, N. Tsagarakis, J. S. Victor, F. Becchi, G. Sandini, and G. Metta, "The design of the iCub humanoid robot," International journal of humanoid robotics, vol. 9, no. 04, p. 1250027, 2012.

[6] D. Shah, G. Metta, and A. Parmiggiani, "Workspace analysis and the effect of geometric parameters for parallel mechanisms of the N-UU class [submitted].".

[7] D. Shah, G. Metta, and A. Parmiggiani, "Comparison of workspace analysis for different spherical parallel mechanisms," in Advances in Mechanism Design for Robots - MEDER 2018 [Accepted], (Udine, Italy), International Federation for the Promotion of Mechanism and Machine Science [IFToMM], Springer Series Mechanisms and Machine Science, 2018.

[8] I. B. Sebastien Briot, "Are parallel robots more accurate than serial robots?," Tech. Rep. pp.445-456, Ecole de technologie suprieure, 04 2007.

[9] J.-O. Kim and K. Khosla, "Dexterity measures for design and control of manipulators," in Intelligent Robots and Systems '91. 'Intelligence for Mechanical Systems, Proceedings IROS '91. IEEE/RSJ International Workshop on, pp. 758-763 vol.2, Nov 1991.

[10] M. Ogata and S. Hirose, "Study on ankle mechanism for walking robots: development of 2 d.o.f. coupled drive ankle mechanism with wide motion range," in 2004 IEEE/RSJ International Conference on Intelligent Robots and Systems (IROS) (IEEE Cat. No.04CH37566), vol. 4, pp. 3201-3206 vol.4, Sept 2004. 
[11] K. Ueda, H. Yamada, H. Ishida, and S. Hirose, "Design of large motion range and heavy duty 2-DOF spherical parallel wrist mechanism," Journal of Robotics and Mechatronics, vol. 25, pp. 294-305, Apr. 2013.

[12] C. Gosselin and J. Angeles, "A global performance index for the kinematic optimization of robotic manipulators," vol. 113, pp. 220 226, 091991.

[13] J. K. Salisbury and J. J. Craig, "Articulated hands: Force control and kinematic issues," The International Journal of Robotics Research, vol. 1 , no. 1 , pp. 4-17, 1982.

[14] R. Storn and K. Price, "Differential evolution - a simple and efficient heuristic for global optimization over continuous spaces," Journal of Global Optimization, vol. 11, pp. 341-359, Dec 1997.

[15] T.-C. Chiang, C.-N. Chen, and Y.-C. Lin, "Parameter control mechanisms in differential evolution: A tutorial review and taxonomy," in 2013 IEEE Symposium on Differential Evolution (SDE), pp. 1-8, April 2013.

[16] G. van Rossum, "Python tutorial," Tech. Rep. CS-R9526, Centrum voor Wiskunde en Informatica (CWI), Amsterdam, May 1995.

[17] M. D. Mckay, R. Beckman, and W. Conover, "A comparison of three methods for selecting vales of input variables in the analysis of output from a computer code," vol. 21, pp. 239-245, 051979. 